\title{
Conservation laws in quantum noninvasive measurements
}

\author{
Stanisław Sołtan, ${ }^{1}$ Mateusz Frączak, ${ }^{1}$ Wolfgang Belzig $\odot,{ }^{2, *}$ and Adam Bednorz $\odot^{1}$ \\ ${ }^{1}$ Faculty of Physics, University of Warsaw, ulica Pasteura 5, 02-093 Warsaw, Poland \\ ${ }^{2}$ Fachbereich Physik, Universität Konstanz, D-78457 Konstanz, Germany
}

(Received 26 July 2019; accepted 1 March 2021; published 17 March 2021)

\begin{abstract}
Conservation principles are essential to describe and quantify dynamical processes in all areas of physics. Classically, a conservation law holds because the description of reality can be considered independent of an observation (measurement). In quantum mechanics, however, invasive observations change quantities drastically, irrespective of any classical conservation law. One may hope to overcome this nonconservation by performing a weak, almost noninvasive measurement. Interestingly, we find that the nonconservation is manifest even in weakly measured correlations if some of the other observables do not commute with the conserved quantity. Our observations show that conservation laws in quantum mechanics should be considered in their specific measurement context. We provide experimentally feasible examples to observe the apparent nonconservation of energy and angular momentum.
\end{abstract}

DOI: 10.1103/PhysRevResearch.3.013247

\section{INTRODUCTION}

Conserved quantities play an important role in both classical and quantum mechanics. According to the classical Noether theorem, the invariance of the dynamics of a system under specific transformations [1] implies the conservation of certain quantities: Translation symmetry in time and space results in energy and momentum conservation, respectively, and rotational symmetry in angular momentum conservation and gauge invariance in a conserved charge. In quantum mechanics, the observables (in the Heisenberg picture) are time independent when they commute with the Hamiltonian. Furthermore, some conserved quantities, like the total charge, commute also with all observables. We shall call them superconserved. Classically, all conserved quantities are also superconserved. In high energy nomenclature, the former are known as on-shell conserved, whereas the latter are called off-shell conserved [2]. The concept of superconservation is closely related to the superselection rule, which constitutes an additional postulate that the set of observables is restricted to those commuting with the superconserved operators [3].

Conservation principles become less obvious when one tries to verify them experimentally. While an ideal classical measurement will keep the relevant quantities unchanged, neither a nonideal classical nor any quantum measurement will necessarily reflect the conservation exactly. Even the smallest interaction between the system and the measuring device

\footnotetext{
*Wolfgang.Belzig@uni-konstanz.de

Published by the American Physical Society under the terms of the Creative Commons Attribution 4.0 International license. Further distribution of this work must maintain attribution to the author(s) and the published article's title, journal citation, and DOI.
}

(detector) may involve a transfer of the conserved quantity. The system might become a coherent superposition of states with different values of a conserved quantity (e.g., energy), or in the case of a superconserved quantity, an incoherent mixture (e.g., charge). The problem of proper modeling of the measurement of quantities incompatible with conserved ones was noticed long ago by Wigner, Araki, and Yanase (WAY) [4-6], later discussed in the context of consistent histories [7], modular values [8], and the quantum clock [9]. The generation, measurement, and control of quantum conserved quantities, in particular, angular momentum, has become interesting recently, both experimentally and theoretically [10-12]. Measurements incompatible with energy lead to thermodynamic cost [13-15].

The quantum objectivity is one aspect of the general concern of Einstein [16] and Mermin [17]—if the (quantum) moon exists when nobody looks. The randomness of quantum mechanics does not exclude objective reality [18]. Here, we assume that objective observations should be noninvasive, i.e., leaving the probed system unchanged. Unfortunately, unlike the classical case, the fundamental uncertainty prevents a completely noninvasive measurement in quantum mechanics [19]. Hence, the only remaining possibility seems to be to consider the limit of weak measurements, which are almost noninvasive. The objectivity based on weak measurements can lead to unexpected results, such as weak values [20] or the violation of the Leggett-Garg inequality [21-23]. Unlike the standard projection, which is highly invasive, the extraction of objective values from weak measurements requires a special protocol involving the subtraction of a large detection noise. Therefore, such objectivity is debatable [24,25]. In our opinion, weak quantum measurements are the closest counterparts of classical measurements [26], so they are prime candidates to define objective reality and, consequently, conservation principles are expected to hold in systems with an appropriate invariance. 


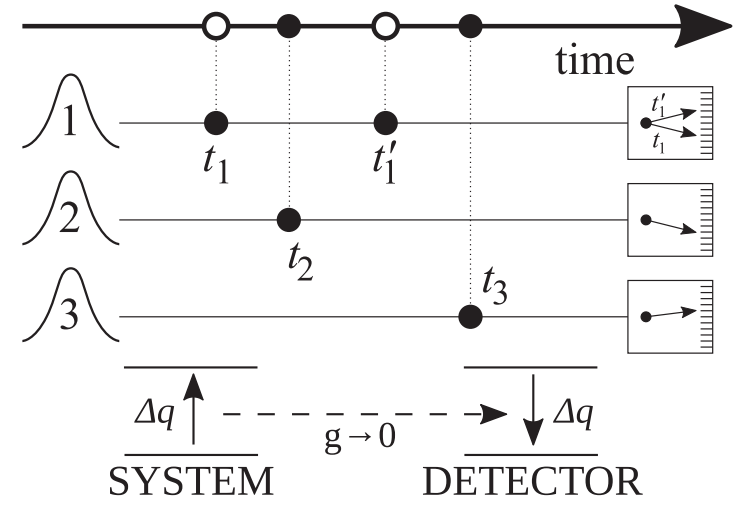

FIG. 1. (Top) Three weak measurements. The detectors are initially independent and couple instantly to the system at $t_{1}$ (or $\left.t_{1}^{\prime}\right), t_{2}$, and $t_{3}$. The conserved quantity is measured (empty circles) either at time $t_{1}<t_{2}$ or $t_{1}^{\prime}>t_{2}$. The outcomes 1 and $1^{\prime}$ inferred from the three-point correlator might differ, even for a conserved quantity in the quantum case. (Bottom) Failure of conservation in the weak measurement. The transfer $\Delta q$ of the conserved quantity $q$ between the system and detector does not scale with the measurement strength $g$.

In this paper, we show that for quantum measurements in the weak limit superconservation holds, but quantities such as energy, momentum, and angular momentum apparently violate conservation even if an appropriate symmetry results in classical conservation law. The violation of conservation appears in third-order time correlations as we illustrate in simple model systems (Fig. 1). The violation is caused by (at least two) other observables that are not commuting with the conserved one. We formulate an operational criterion to witness the violation of a conservation principle and discuss when it is satisfied. Then, we propose a feasible experiment probing position and magnetic moment of a charge in a circular trap. Lastly, considering an imperfect conservation or measurement of the quantity, we develop then a Leggett-Garg-type test of objective realism.

\section{SUPERCONSERVATION}

The physical Hermitian quantity $\hat{Q}$ defined within the system is conserved when $[\hat{H}, \hat{Q}]=0$ for the system's Hamiltonian $\hat{H}$. The quantity $\hat{Q}$ can be superconserved if there exists a set $\mathcal{A}$ of allowed Hermitian observables such that $[\hat{A}, \hat{Q}]=0$ for every $\hat{A} \in \mathcal{A}$. In principle, one could make every conserved quantity superconserved by a proper choice of the set $\mathcal{A}$. However, for instance, for a component of angular momentum, we would have to exclude position and momentum or even other components of angular momentum. Instead, we will distinguish quantities that are conserved but not superconserved by allowing measurement of observables not commuting with them. An example of a superconserved quantity is the total electric charge, while the set of observables and possible initial state density matrices is restricted to those that do not change the charge. This is also known as superselection rule. Whether this rule is an axiom or a practical assumption, depending on the Hamiltonian considered, is a matter of debate [3], because one can, in principle, imagine dynamics without, e.g., charge superselection. Nevertheless, here we treat superselection and superconservation as an axiom for certain quantities, like charge. Let us assume the decomposition of a superconserved quantity $\hat{Q}=\sum_{q} q \hat{P}_{q}$, where $\hat{P}_{q}$ are (mutually commuting) projections onto the eigenspace of the value $q$ (i.e., $\hat{Q}\left|\psi_{q}\right\rangle=q\left|\psi_{q}\right\rangle$ ). Now, the superselection postulate says that the state of the system $\hat{\rho}$ is always an incoherent mixture $\sum_{q} \hat{P}_{q} \hat{\rho} \hat{P}_{q}$, if $\hat{Q}$ is superconserved. Then, the projective measurement of $\hat{A}$ will not alter the $q$-eigenspace as there exists a decomposition $\hat{A}=\sum_{q, a} a \hat{P}_{q a}$ with $\hat{P}_{q a}$ being the projection onto the joint eigenspace of $\hat{Q}$ and $\hat{A}$ with respective eigenvalues $q$ and $a$. For instance, if the initial state is already a $q$-eigenstate then it will remain such an eigenstate after the projection. For general measurements, positive operator-valued measures (POVM), represented by Kraus operators $\hat{K}_{c}$ (the index $c$ can represent an eigenvalue of $\hat{A}, \hat{Q}$, or both, but in general it can be arbitrary) such that $\sum_{c} \hat{K}_{c}^{\dagger} \hat{K}_{c}=\hat{1}$, the state $\hat{\rho}$ will collapse to $\hat{\rho}_{c}=\hat{K}_{c} \hat{\rho} \hat{K}_{c}^{\dagger}$, normalized by the probability $\operatorname{Tr} \hat{\rho}_{c}$. In principle, $\hat{K}_{c}$ can act within $q$-eigenspaces, i.e., $c=q a$ and $\hat{K}_{q a}=\hat{P}_{q} \hat{K}_{a} \hat{P}_{q}$. In the most general case, the superconserving Kraus operator reads

$$
\hat{K}_{q^{\prime} a q}=\hat{P}_{q^{\prime}} \hat{K}_{a} \hat{P}_{q} .
$$

It means that the superconserved value can change but the system remains an incoherent mixture of $q$-eigenstates. This applies, e.g., to a charge measurement in a quantum dot (which is superconserved), where the charge can leak out into an incoherent bath. The (normally) conserved quantities do not impose any additional postulates, so the state can be a coherent superposition of the states of different values of energy, angular momentum, etc. A projective measurement of $\hat{A}$, which does not commute with $Q$ is enough to turn a $q$-eigenstate into a superposition. Now, if we try to postulate a POVM with superconserving Kraus operators then the actually measured operator involves a linear combination of $\sum_{q q^{\prime}} \hat{P}_{q} \hat{K}_{a}^{\dagger} \hat{P}_{q^{\prime}} \hat{K}_{a} \hat{P}_{q}$, so it must commute with $\hat{Q}$, which would become superconserved. This is a modern version of the WAY theorem [4-6] in that the measurement of $\hat{A}=\sum_{a} a \hat{K}_{a}^{\dagger} \hat{K}_{a}$ not commuting with $\hat{Q}$, cannot consist of only $\hat{K}_{q^{\prime} a q}$ defined above with $q$-eigenspaces of $\hat{Q}$. Such a formulation is simpler than the original WAY theorem, as is does not need the discussion of an auxiliary detector. On the other hand, both approaches are equivalent because of the Naimark theorem [27].

The unavoidability of coherent superpositions of only conserved values is the key problem considered here.

\section{WEAK MEASUREMENTS AND OBJECTIVE REALISM}

Strong projections are highly invasive, i.e., $\hat{\rho} \rightarrow \sum_{P} \hat{P} \hat{\rho} \hat{P}$ changes the state $\rho$ very much. On the other hand, unlike classical physics, quantum mechanics does not offer completely noninvasive measurements. The only possibility is weak measurement [20], where we apply Kraus operators

$$
\hat{K}_{g}(a)=(2 g / \pi)^{1 / 4} \exp \left(-g(\hat{A}-a)^{2}\right),
$$

with the measurement strength $g \rightarrow 0_{+}$so that the state almost does not change

$$
\hat{\rho} \rightarrow \int d a \hat{K}(a) \hat{\rho} \hat{K}(a) \approx \hat{\rho}-g[\hat{A},[\hat{A}, \hat{\rho}]] / 2 .
$$


The actually measured probability $p^{\prime}(a)=\operatorname{Tr} \hat{K}(a) \hat{\rho} \hat{K}^{\dagger}(a)$ of the outcome $a$ at the state $\hat{\rho}$ has a form of convolution

$$
\begin{aligned}
p^{\prime}(a) & =\int D(a-A) p(A) d A, \\
p(A) & =\langle\delta(A-\hat{A})\rangle=\operatorname{Tr} \delta(A-\hat{A}) \hat{\rho},
\end{aligned}
$$

with the dominating detection noise $D(a)=\sqrt{2 g / \pi} e^{-2 g a^{2}}$, and with $\left\langle a^{2}\right\rangle_{D}=1 / 4 g$, diverging for $g \rightarrow 0$. The quantity $p(A)$ is the probability of the outcome $A$ in the case of a strong, projective measurement $g \rightarrow \infty\left(p(A)=\lim _{g \rightarrow \infty} p^{\prime}(A)\right)$, to which the noise $D$ is added. Therefore, we can expect that $p(A)$ is in fact the probability that the quantity $\hat{A}$ has objectively the value $A$. Unfortunately, such an idea fails in sequential measurements, as shown already [20], because $p(A, B)$ can be negative when measuring first $\hat{A}$ and then $\hat{B}$, such that $[\hat{A}, \hat{B}] \neq 0$. The original concept $[20]$ involved postselection, i.e., the last measurement is strong, not weak, and the conditional probability $p(A, B) / p(B)$ is considered. However, the strength of the last measurement is irrelevant, as the system is not touched any more. In our approach, all measurements, including the last one, can be assumed weak.

We shall discuss the problem of a negative $p$ in Sec. VI. Nevertheless, $p(A, B, \ldots)$ is well defined in the limit $g \rightarrow 0$ and we can probe it, hence, assuming that it reflects a property of the system. Note that this construction is still correct in the superconserved case because $\hat{A}, \hat{K}_{a}$, and the state $\hat{\rho}$ is commuting with $\hat{Q}$ so $\hat{K}_{a}$ splits into a simple sum of $\hat{K}_{q a}$. The actual form of $\hat{K}_{a}$ can be different but the outcome is almost independent in the limit $g \rightarrow 0$. In the lowest order, we can also neglect all $\hat{K}_{q^{\prime} a q}$. In the $g \rightarrow 0$ limit, the $n$ th-order correlation of a sequence of measurements $\hat{A}, \hat{B}, \hat{C}$, and $\hat{D}$ with respect to $p$ (or also $p^{\prime}$ if the quantities are different) reads [26,28,29]

$$
\begin{gathered}
\langle a(t)\rangle=\langle\hat{A}(t)\rangle, \\
\left\langle a\left(t_{1}\right) b\left(t_{2}\right)\right\rangle=\left\langle\left\{\hat{A}\left(t_{1}\right), \hat{B}\left(t_{2}\right)\right\}\right\rangle / 2, \\
\left\langle a\left(t_{1}\right) b\left(t_{2}\right) c\left(t_{3}\right)\right\rangle=\left\langle\left\{\hat{A}\left(t_{1}\right),\left\{\hat{B}\left(t_{2}\right), \hat{C}\left(t_{3}\right)\right\}\right\}\right\rangle / 4, \\
\left\langle a\left(t_{1}\right) b\left(t_{2}\right) c\left(t_{3}\right) d\left(t_{4}\right)\right\rangle \\
=\left\langle\left\{\hat{A}\left(t_{1}\right),\left\{\hat{B}\left(t_{2}\right),\left\{\hat{C}\left(t_{3}\right), \hat{D}\left(t_{4}\right)\right\}\right\}\right\}\right\rangle / 8,
\end{gathered}
$$

for $t_{1}<t_{2}<t_{3}<t_{4}$ with the anticommutator $\{\hat{A}, \hat{B}\}=\hat{A} \hat{B}+$ $\hat{B} \hat{A}$ and quantum averages $\langle\hat{X}\rangle=\operatorname{Tr} \hat{X} \hat{\rho}$.

At this stage, we would like to note that the classical counterpart of this protocol replaces the anticommutators like $\{\hat{A}, \hat{B}\}$ by simple products of phase-space functions $A(q, p)$ and $B(q, p)$. The invasiveness (3) can be reduced to zero and the time order of observables is irrelevant. For a more detailed analysis of classical-to-quantum correspondence, we refer the reader to Ref. [26].

\section{CONSERVATION IN WEAK MEASUREMENTS}

The conservation means that the measurable correlations (7) involving the conserved quantity $q(t)$ corresponding to $\hat{Q}(t)=\hat{Q}$ will not depend on $t$. It is true at the single average, where $\langle q(t)\rangle=\langle\hat{Q}\rangle$. Interestingly, also for second-order correlations, the order of measurements has no influence on the result since $\left\langle q\left(t_{1}\right) a\left(t_{2}\right)\right\rangle=\left\langle\left\{Q, A\left(t_{2}\right)\right\}\right\rangle / 2$ is independent of $t_{1}$. However, the situation changes for three consecutive measurements (see Fig. 1), since in the last line of (7) the time order of operators matters, which has also been demonstrated experimentally [30]. Considering the difference of two measurement sequences $Q \rightarrow A \rightarrow B$ and $A \rightarrow Q \rightarrow B$, we obtain the jump (which is absent in perfectly noninvasive classical measurements [26])

$$
\begin{aligned}
& \left\langle\left\{\hat{Q},\left\{\hat{A}\left(t_{2}\right), \hat{B}\left(t_{3}\right)\right\}\right\}-\left\{\hat{A}\left(t_{2}\right),\left\{\hat{Q}, \hat{B}\left(t_{3}\right)\right\}\right\}\right\rangle \\
& \quad=\left\langle\left[\left[\hat{Q}, \hat{A}\left(t_{2}\right)\right], \hat{B}\left(t_{3}\right)\right]\right\rangle \equiv 4\left\langle\Delta q a\left(t_{2}\right) b\left(t_{3}\right)\right\rangle .
\end{aligned}
$$

This quantity will show up as jump $\Delta q=q\left(t_{1}\right)-q\left(t_{2}\right)$ at $t_{1}=t_{2}$ when measuring $\left\langle q\left(t_{1}\right) a\left(t_{2}\right) b\left(t_{3}\right)\right\rangle$. The jump will be nonzero for $Q$ not commuting with $A$ and $B$. Obviously, for superconserved quantities $Q$ (commuting with every measurable observable), the jump is absent. The violation of the conservation principle is caused by the measurement of $\hat{A}$, not commuting with $\hat{Q}$, which allows transitions between spaces of different $q$ with the jump size $\Delta q$ not scaled by the measurement strength $g$ (see Fig. 1). This difference is transferred to the detector, assuming that the total quantity (of the system and detector) is conserved regardless of the systemdetector interaction. This observation can be compared to the WAY theorem, which applies to projective or general measurements. Here, we have shown that even taking the special limit of noninvasive measurement, the noncommuting quantity causes a jump in third-order (and higher) correlations. We can call it weak-WAY theorem, as both the input (the special construction of $g$-dependent measurements) and the output (correlations) are based on weak measurements. Note that imposing the condition that the jump (9) vanishes, equivalent conservation of $\hat{Q}$ at the level of third-order correlation for an arbitrary state $\hat{\rho}$ (allowed by superselection rules, if any apply), namely,

$$
[[\hat{Q}, \hat{A}], \hat{B}]=0,
$$

for all allowed observables $\hat{A}$ and $\hat{B}$ suffices to keep conservation also at all higher-order correlations. Then, $\hat{Q}$ is not necessarily superconserved, it can commute with observables to identity, like momentum and position. This subtle difference between the weak-WAY and the traditional WAY theorem in sketched in Fig. 2.

As an example, we can take the basic two-level system $(| \pm\rangle$ basis) with the Hamiltonian $\hat{H}=\hat{Q}=\hbar \omega|+\rangle\langle+|$ and $\hat{A}=\hat{B}=\hat{X}=|+\rangle\langle-|+|-\rangle\langle+|$. Then, with $\omega>0$ the ground state is $|-\rangle$ and the third-order correlation $\left\langle h\left(t_{1}\right) x\left(t_{2}\right) x\left(t_{3}\right)\right\rangle$ for the ground state for $t_{3}>t_{1,2}$ reads $\hbar \omega(1-$ $\left.\theta\left(t_{2}-t_{1}\right)\right) \cos \left(\omega\left(t_{2}-t_{3}\right)\right) / 2$. The jump is $\langle\Delta h x(0) x(\tau)\rangle=$ $\hbar \omega \cos (\omega \tau) / 2$ for $\Delta h=h\left(0_{-}\right)-h\left(0_{+}\right)$. The result can be generalized to a thermodynamical ensemble with a finite temperature $T$ and reads (see Appendix A)

$$
\langle\Delta h x(0) x(\tau)\rangle=\hbar \omega \cos (\omega \tau) \tanh (\hbar \omega / 2 k T) / 2 .
$$

For increasing temperature, the jump diminishes as illustrated in Fig. 3.

Another basic example is the harmonic oscillator with $\hat{H}=\hat{Q}=\hbar \omega \hat{a}^{\dagger} \hat{a}$ with $\left[\hat{a}, \hat{a}^{\dagger}\right]=1$. Taking the dimensionless position $\sqrt{2} \hat{X}=\hat{a}^{\dagger}+\hat{a}=\hat{A}=\hat{B}$, we find for the jump 


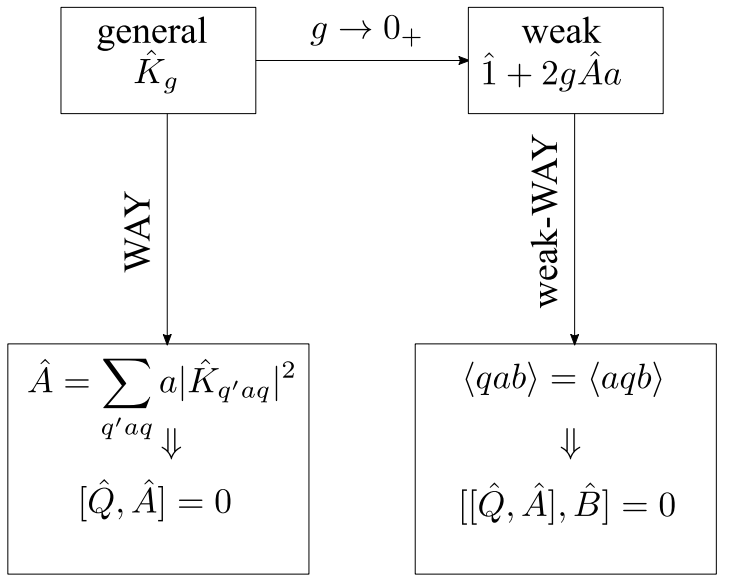

FIG. 2. The difference between the WAY and the weak-WAY theorem. The former applies to general measurement and shows that the lack of coherence between eigenstates of $\hat{Q}$ in the Kraus operators (1) leads to superconservation of the measured quantity. The latter applies to weak measurements (2), leading to a weaker condition for the observed conservation.

$\langle\Delta h x(0) x(t)\rangle=-\hbar \omega \cos (\omega t) / 4$, independent of the state of the system (see Appendix A). As illustrated in Fig. 3, the jump becomes unobservable at high temperatures since the average energy $\langle h\rangle=\hbar \omega /[\exp (\hbar \omega / k T)-1]$ increases with temperature.

The previously discussed very simple examples illustrate the fundamental finding of our paper. If one tries to verify the conservation of energy while measuring another observable that is not commuting with the Hamiltonian, it is possible to find a violation of the energy conservation. It constitutes a pure quantum effect since it vanishes at high temperature, where the classically expected conservation holds. One could object that performing a series of measurements already breaks time-translational symmetry and, therefore, the total energy is not conserved. However, one can keep the time symmetry by replacing the detector-system interaction by a clock-based detection scheme [9] (see Fig. 4). The total

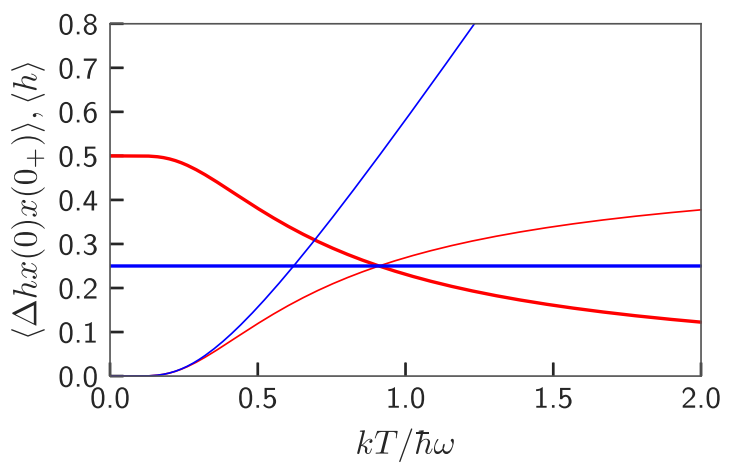

FIG. 3. The nonconserving jump for $\tau=0_{+}$(thick lines) compared to the average energy (thin lines) for the two-level system with level spacing $\hbar \omega$ (red) and the harmonic oscillator with eigenfrequency $\omega$ (blue). At high temperatures, the jump becomes unobservable and the classical conservation is restored. All quantities are normalized to $\hbar \omega$.

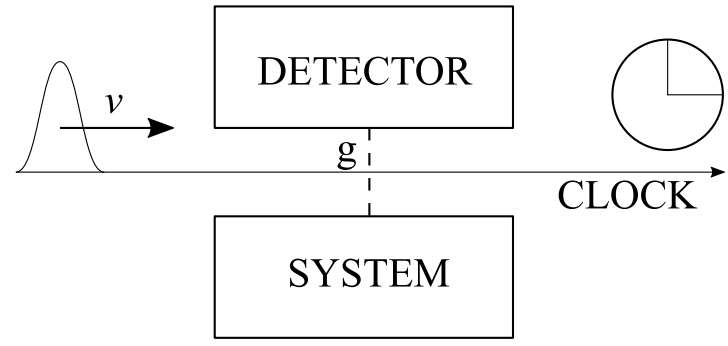

FIG. 4. Detection model based on a clock. The clock is a localized particle traveling with a constant speed $v$. The interaction between the detector and system takes place only when the clock is passing the interaction point.

Hamiltonian reads

$$
\hat{H}+\hat{H}_{x}+\hat{H}_{z}+\hat{H}_{I},
$$

where $\hat{H}$ is the system's part, $\hat{H}_{x}$ is the detector's part, $\hat{H}_{z}$ is the clock's part, and $\hat{H}_{I}$ is the interaction between the clock, the system, and detector. Each part is time-independent so the time-translation symmetry is preserved. Both the detector and the clock can be represented by single real variables, $x$ and $z$. Now, to measure the system's $\hat{A}$ at time $t_{1}$, we set $\hat{H}_{x}=0$ and

$$
\hat{H}_{z}=v \hat{p}_{z}, \quad \hat{H}_{I}=g \hat{A} \delta(\hat{z}) \hat{p}_{x},
$$

where $\hat{p}_{x, z}$ are conjugate (momenta), i.e., $\hat{p}_{x}=-i \hbar \partial / \partial x$ and $g \rightarrow 0$ is a weak coupling constant. The initial state (at $t=0$ ) reads $\hat{\rho} \hat{\rho}_{x} \hat{\rho}_{z}$, where both $\hat{\rho}_{x, z}=\left|\psi_{x, z}\right\rangle\left\langle\psi_{x, z}\right|$ are taken as Gaussian states

$$
\begin{aligned}
& \psi_{z}(z)=(2 \pi \sigma)^{-1 / 4} \exp \left(-\left(z+v t_{1}\right)^{2} / 4 \sigma\right), \\
& \psi_{x}(x)=(\pi / 2)^{-1 / 4} \exp \left(-x^{2}\right),
\end{aligned}
$$

respectively. For small $g$ and $\sigma$, the interaction effectively occurs at time $t=t_{1}$ and, in the end (after the clock decouples the system and the detector again) to lowest order we find (see details in Appendix B)

$$
\langle x\rangle \simeq g\langle\hat{A}\rangle=g\left\langle a\left(t_{1}\right)\right\rangle .
$$

For sequential measurements, one simply adds more independent detectors and clocks, obtaining in the lowest order of $g$

$$
\begin{aligned}
\left\langle x_{A} x_{B}\right\rangle & \simeq g^{2}\left\langle a\left(t_{1}\right) b\left(t_{2}\right)\right\rangle, \\
\left\langle x_{A} x_{B} x_{C}\right\rangle & \simeq g^{3}\left\langle a\left(t_{1}\right) b\left(t_{2}\right) c\left(t_{3}\right)\right\rangle, \\
\left\langle x_{A} x_{B} x_{C} x_{D}\right\rangle & \simeq g^{4}\left\langle a\left(t_{1}\right) b\left(t_{2}\right) c\left(t_{3}\right) d\left(t_{4}\right)\right\rangle,
\end{aligned}
$$

with the right-hand sides are given by the quantum expressions (7).

Although the above detection model is based on timeinvariant dynamics, the initial state of the clock spoils the symmetry. The time-invariant state would require a constant flow of particles or field at a constant velocity, so that the position on the tape imprints time of measurement (see [31] for detailed construction). However, such a constant interaction between the detector (clock) and the system leads to a backaction and makes the measurement invasiveness growing with time, which needs to be reduced by additional resources, e.g., additional coupling to a heat bath.

In order to show that the nonconservation can also occur independently from the time-translation asymmetry present 


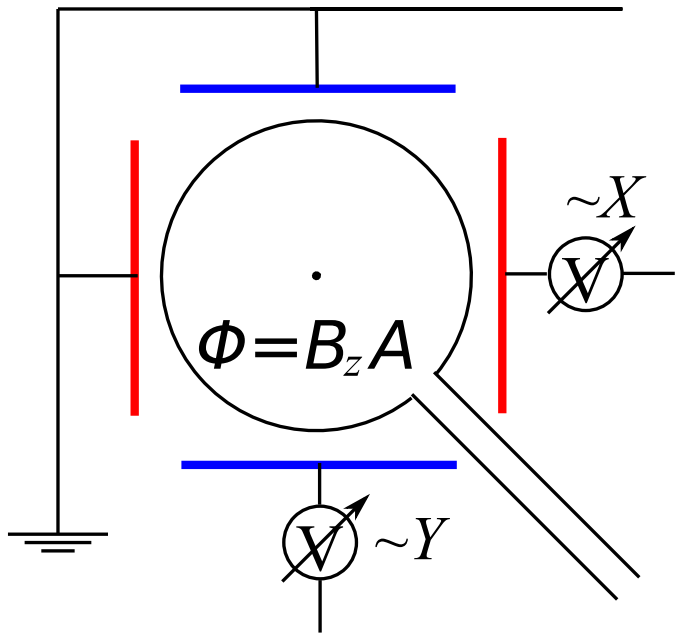

FIG. 5. A trap for a charged particle invariant under rotation about $z$ axis with a detector weakly coupled to angular momentum $L_{z}$ and the position in the $x-y$ plane detected by appropriate capacitors. The magnetic moment of the particle can be measured by a sensitive magnetometer, e.g., a SQUID.

either intrinsically or induced by a quantum clock, one can look at other quantities that are conserved, e.g., due to spatial symmetries. As an example, we will use one component of the angular momentum in a rotationally invariant system in the following.

\section{ANGULAR MOMENTUM CONSERVATION}

We propose an experiment to demonstrate the failure of the conservation principle for angular momentum in thirdorder correlations in weak measurements. Instead of energy, we consider one component of angular momentum, say $\hat{L}_{z}=$ $\hat{X} \hat{P}_{y}-\hat{Y} \hat{P}_{x}$, which can be measured in principle by a sensitive magnetometer (e.g., a superconducting quantum interferometer device). The other two observables will be the particle's positions $\hat{X}$ and $\hat{Y}$, with the readouts $x$ and $y$ respectively, which can be measured, e.g., by the voltage of a capacitor depending linearly on $x$ and $y$ for small changes in position (see the setup sketch in Fig. 5). The two positions $x$ and $y$ will be measured at times $t_{2}$ and $t_{3}$, respectively.

The quantity of matter is $\left\langle l_{z}\left(t_{1}\right) x\left(t_{2}\right) y\left(t_{3}\right)\right\rangle$. Suppose the particle is in a harmonic trap rotationally invariant about the $z$ axis. The $x y$ part of the trap Hamiltonian reads $\hat{H}_{\perp}=$ $\hbar \omega\left(\hat{a}_{x}^{\dagger} \hat{a}_{x}+\hat{a}_{y}^{\dagger} \hat{a}_{y}\right)$, with $\left[\hat{a}_{x, y}, \hat{a}_{x, y}^{\dagger}\right]=1,\left[\hat{a}_{x}, \hat{a}_{y}\right]=\left[\hat{a}_{x}^{\dagger}, \hat{a}_{y}\right]=$ 0 . Then $\sqrt{2} \hat{X}=\hat{a}_{x}^{\dagger}+\hat{a}_{x}$ and $\sqrt{2} \hat{P}_{x} / i \hbar=\hat{a}_{x}^{\dagger}-\hat{a}_{x}$ (rescaled by a length unit), similarly for $y$, and $\hat{L}_{z}=i \hbar\left(\hat{a}_{x} \hat{a}_{y}^{\dagger}-\right.$ $\left.\hat{a}_{y} \hat{a}_{x}^{\dagger}\right)$. In the ground state $|0\rangle$, we have $\hat{L}_{z}|0\rangle=0$, so only $\left\langle\hat{Y}\left(t_{3}\right) \hat{L}_{z}\left(t_{1}\right) \hat{X}\left(t_{2}\right)\right\rangle$ and $\left\langle\hat{X}\left(t_{2}\right) \hat{L}_{z}\left(t_{1}\right) \hat{Y}\left(t_{3}\right)\right\rangle$ contribute in (7). These terms can appear only when $t_{2}<t_{1}$ or $t_{3}<t_{1}$. We find

$$
\begin{aligned}
\left\langle l_{z}\left(t_{1}\right) x\left(t_{2}\right) y\left(t_{3}\right)\right\rangle= & \left(1-\theta\left(t_{2}-t_{1}\right) \theta\left(t_{3}-t_{1}\right)\right) \\
& \times\left\langle\hat{X}\left(t_{2}\right) \hat{L}_{z}\left(t_{1}\right) \hat{Y}\left(t_{3}\right)+\hat{Y}\left(t_{3}\right) \hat{L}_{z}\left(t_{1}\right) \hat{X}\left(t_{2}\right)\right\rangle / 4 \\
= & \left(1-\theta\left(t_{2}-t_{1}\right) \theta\left(t_{3}-t_{1}\right)\right) \hbar \sin \omega\left(t_{2}-t_{3}\right) / 4 .
\end{aligned}
$$

The jump is therefore given by

$$
\left\langle\Delta l_{z} x\left(t_{2}\right) y\left(t_{3}\right)\right\rangle_{0}=\hbar \sin \left[\omega\left(t_{2}-t_{3}\right)\right] / 4
$$

and is again state-independent as in the case of the harmonic oscillator. It illustrates that the angular momentum conservation is violated by this experiment. At finite temperature $T$ for $t_{1}<t_{2,3}$, the correlator $\left\langle l_{z} x\left(t_{2}\right) y\left(t_{3}\right)\right\rangle=\hbar \sin \left[\omega\left(t_{2}-\right.\right.$ $\left.\left.t_{3}\right)\right] / 4 \sinh ^{2}\left(\hbar \omega / 2 k_{B} T\right)$ increases with temperature and makes the (temperature-independent) jump unobservable.

Since in this setup the detectors are coupled permanently, a frequency-domain measurement might be more appropriate. In the frequency domain, the observables are $A(\alpha)=$ $\int d t e^{i \alpha t} A(t)$. Taking all our previous arguments to frequency domain, the conservation of a quantity $\hat{Q}(\alpha)$ means that correlators vanish for $\alpha \neq 0$. Interestingly, transforming to frequency domain, we find at zero temperature and for $\gamma, \alpha, \beta \neq 0$ that

$$
\left\langle l_{z}(\gamma) x(\alpha) y(\beta)\right\rangle=\frac{i \pi \hbar \omega(\beta-\alpha) \delta(\gamma+\alpha+\beta)}{2\left(\alpha^{2}-\omega^{2}\right)\left(\beta^{2}-\omega^{2}\right)} .
$$

The conservation principle for angular momentum is violated by (19) because it is nonzero. Hence, either by time- or frequency-resolved measurements, one should see experimentally the nonconservation of angular momentum.

To realize a time-resolved measurement, we suggest to test the angular momentum conservation with a charge moving inside a round tube along $z$ direction, similar to the recent test of the order of measurements [30]. In the simplest model, take $\hat{H}=\hat{H}_{z}+\hat{H}_{\perp}$ and we keep the same harmonic potential in the $x-y$ plane as above and add some $\hat{H}_{z}=v \hat{p}_{z}$ with velocity $v$ (like the clock in the previous section). Preparing a wavepacket as a product of the ground state of $\hat{H}_{\perp}$ and $\psi(z)$ of sufficiently short width, we can measure essentially the same quantity (17) by placing a sequence of weak detectors along the tube (see Fig. 6). The angular momentum can be measured by the current signature in the coil, like in the recent experiment [10]. We simplify the coil-electron beam interaction to $\lambda(z) \hat{I} \hat{L}_{z}$ where $\lambda$ is only nonzero inside the coil. Similarly, the measurement of $x$ and $y$ can be modeled by local capacitive couplings. In this way, the measurement times are translated into position according to $t=z / v$, similar to the time-invariant energy detection in the previous section. The jump (18) can then be detected by placing the coil at two different positions (see Fig. 6).

Regarding the rotational invariance of the system, detection of $X$ and $Y$ can be performed by the detector-system coupling

$$
\hat{H}_{I}=g \delta(\hat{z})\left(\hat{X} \hat{p}_{x D}+\hat{Y} \hat{p}_{y D}\right)
$$

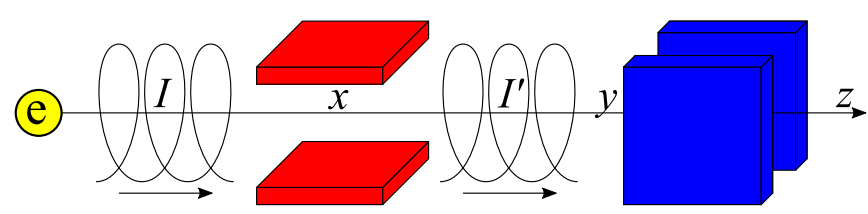

FIG. 6. A charged particle (e.g., electron) goes along the tube with angular momentum measured by current in either of two coils, $I$ or $I^{\prime}$ while the position in $x$ and $y$ direction is measured by two perpendicular capacitors. 
with the initial state $\hat{\rho} \hat{\rho}_{x, y}^{D}$ and the detector's state $\hat{\rho}_{x, y}^{D}=$ $|\psi\rangle\langle\psi|$,

$$
\psi\left(x_{D}, y_{D}\right)=(\pi / 2)^{-1 / 2} \exp \left(-\left(x_{D}^{2}+y_{D}^{2}\right)\right) .
$$

Then, both the interaction and the initial state of the system and detector are rotationally invariant so the total angular momentum is conserved. Only the readout, either $x$ or $y$ of the detector, prefers one direction, i.e.,

$$
\left\langle x_{D}\right\rangle \simeq g\langle x\rangle,
$$

with straightforward generalization to sequential measurements like (16).

\section{LEGGETT-GARG INEQUALITIES}

The above proposals face some practical challenges. The velocity $v$ should be sufficiently high to ignore decoherence effects, e.g., due to coupling to a thermal environment. The decoherence could be modeled by Lindblad-type terms added to the Hamiltonian dynamics of the density matrix. The test of conservation makes sense only for times/frequencies within the coherence timescale. Any observable roughly tracking the charge in two perpendicular directions will suffice. The tube may be not perfectly harmonic or not homogeneous in the $z$ direction, and $\hat{L}_{z}$ can be only approximately conserved or imprecisely measured. To quantify these considerations, we will now develop a Leggett-Garg-type test [21]. Let us consider the measurement of four observables: $q=q\left(t_{1}\right), q^{\prime}=q\left(t_{1}^{\prime}\right)$, $x=x\left(t_{2}\right)$, and $y=y\left(t_{3}\right)$ with $q$ being an approximate value of the conserved quantity and $t_{1}<t_{2}<t_{1}^{\prime}<t_{3}$. Here, $q, q^{\prime}$ can correspond to angular momentum $l_{z}$, while $x, y$ are the lateral positions in the test presented in the previous section. Note that Leggett-Garg-type tests for angular momentum were discussed in a different context $[32,33]$. According to the objective realism assumption, the values of $\left(q, q^{\prime}, x, y\right)$ exist independent of the measurement. If there is a corresponding joint positive probability $p\left(q, q^{\prime}, x, y\right)$, then correlations with respect to $p$ must satisfy the following two CauchyBunyakovsky-Schwarz inequalities:

$$
\begin{aligned}
& \left\langle\left(q-q^{\prime}\right)^{2}\right\rangle_{\rho}\left\langle x^{2} y^{2}\right\rangle_{\rho} \geqslant\left\langle\left(q-q^{\prime}\right) x y\right\rangle_{\rho}^{2}, \\
& \left\langle\left(q-q^{\prime}\right)^{2} y^{2}\right\rangle_{\rho}\left\langle x^{2}\right\rangle_{\rho} \geqslant\left\langle\left(q-q^{\prime}\right) x y\right\rangle_{\rho}^{2} .
\end{aligned}
$$

However, if we test these inequalities using $p$ defined in (4) and quantum correlations (7) then they could be violated. Classically, the measurements of the conserved quantity at two different times should not depend on whether another observable is measured in between and both sides of Eqs. (23) vanish. Using Eqs. (7), the left-hand sides of Eqs. (23) vanish for a perfectly conserved quantity. First, $\left\langle\left(q-q^{\prime}\right)^{2}\right\rangle_{p}=$ 0 because $\hat{Q}(t)=\hat{Q}$ is independent of time. Second, $\langle(q-$ $\left.\left.q^{\prime}\right)^{2} y^{2}\right\rangle_{p}=0$ because in addition $y$ is measured after both $q$ and $q^{\prime}$. On the other hand, the right-hand side of (23) exactly corresponds to the quantum mechanical jump in the third-order correlator as defined in (18). Hence, even if $q$ is not exactly conserved then the left-hand sides can be small enough to violate the inequalities. These violations can be readily tested in the setup suggested in Fig. 6. Note that the inequalities must involve fourth-order moments because of the so-called weak positivity [34] stating that lower moments are insufficient to violate realism for continuous variables.

\section{CONCLUSION}

We have shown that conservation laws in quantum mechanics need to be considered with care since their experimental verification might depend on the measurement context even in the limit of weak measurements. The conservation is violated if extracting objective reality from the weak measurements. It means that either (i) weak measurements cannot be considered noninvasive, or (ii) the conservation laws do not hold in quantum objective realism. Exceptions are superconserved observables, which will be conserved whatever measurement is performed, and more generally observables that satisfy the weak-WAY condition (10). The nonconservation can also be formulated as Leggett-Garg-type test showing the connection to the absence of objective realism in quantum mechanics. In the future, it might be interesting to study more realistic scenarios for quantum measurements taking into account decoherence or more general detectors [35]. Furthermore, one might generalize these findings to more fundamental relativistic field theories [36,37], testing correlations involving components of stress-energy-momentum tensor.

\section{ACKNOWLEDGMENTS}

We thank E. Karimi for fruitful discussion and N. Gisin for bringing the quantum clock to our attention. W.B. gratefully acknowledge the support from Deutsche Forschungsgemeinschaft (DFG, German Research Foundation) through Project-ID 32152442 - SFB 767 and Project-ID 425217212 - SFB 1432.

\section{APPENDIX A: DERIVATION OF CORRELATION JUMPS}

To derive (11), one needs to take the thermal state

$$
\hat{\rho}=Z^{-1}\left(e^{-\hbar \omega / k T}|+\rangle\langle+|+|-\rangle\langle-|\right)
$$

with $Z=1+e^{-\hbar \omega / k T}$ and

$$
\hat{X}(t)=e^{-i \omega t}|+\rangle\left\langle-\left|+e^{i \omega t}\right|-\right\rangle\langle+|
$$

plugged into (9) with $\hat{Q}=\hat{H}=\hbar \omega|+\rangle\langle+|$ and $\hat{A}=\hat{B}=\hat{X}$ with $t_{1}=0_{ \pm}, t_{2}=0$ and $t_{3}=\tau$.

The case of harmonic oscillator can be written in Fock basis $|n\rangle, n=0,1,2, \ldots$ with $\hat{a}|n\rangle=\sqrt{n}|n-1\rangle,\left[\hat{a}, \hat{a}^{\dagger}\right]=1$, $\hat{n}=\hat{a}^{\dagger} \hat{a}=\sum_{n} n|n\rangle\langle n|, \hat{n}| n\rangle=n|n\rangle$, and $\hat{H}=\hat{Q}=\hbar \omega \hat{n}, \hat{X}=$ $\left(\hat{a}+\hat{a}^{\dagger}\right) / \sqrt{2}$. The thermal state

$$
\hat{\rho}=Z^{-1} e^{-\hbar \omega \hat{n} / k T}
$$

with $Z=\left(1-e^{-\hbar \omega / k T}\right)^{-1}$ and

$$
\hat{A}=\hat{B}=\hat{X}(t)=\left(e^{i \omega t} \hat{a}+e^{-i \omega t} \hat{a}^{\dagger}\right) / \sqrt{2} .
$$

The independence of the jump of the state follows from the fact that

$$
\left[\left[\hat{H}, \hat{X}\left(t_{2}\right)\right], \hat{X}\left(t_{3}\right)\right] \propto \hat{1}
$$

because $[\hat{H}, \hat{X}]$ is linear (momentum) in $\hat{a}$ and $\hat{a}^{\dagger}$ and, hence, the outer commutator becomes a $c$-number. 


\section{APPENDIX B: WEAK CORRELATIONS WITH A QUANTUM CLOCK}

A single detector and a single clock are defined by (13) and (14), respectively. The detector position $x$ is measured (projectively) at some time later than the interaction moment $t_{1}$. The average

$$
\langle x\rangle \simeq \operatorname{Tr} \int d t\left[\hat{x}, \hat{H}_{I}(t)\right] \hat{\rho} / i \hbar
$$

in the interaction picture, in the lowest order, according to the decomposition (12). With

$$
\hat{H}_{I}(t)=g \hat{A}(t) \delta(\hat{z}-v t) \hat{p}_{x}
$$

for the initial initial states (14) we have used the identity

$$
[\hat{C} \hat{D}, \hat{\rho}]=\{C,[\hat{D}, \hat{\rho}]\}+\{\hat{D},[\hat{C}, \hat{\rho}]\}
$$

for $\hat{C} \hat{D}=\hat{D} \hat{C}, \quad\left\langle\psi_{x}\left|\left\{\hat{x}, \hat{p}_{x}\right\}\right| \psi_{x}\right\rangle=0 \quad$ (anticommutator), $[\hat{x}, \hat{p}]=i \hbar,\left\langle\psi_{z}|\delta(\hat{z}-v t)| \psi_{z}\right\rangle=\left|\psi_{z}(v t)\right|^{2}$ to get (15). To extend it to the sequential case (16), we do not apply the trace over the system space in (B1) (only over $x$ and $z$ ), getting the matrix

$$
\{\hat{A}(t), \hat{\rho}\} / 2 .
$$

Note that it is Hermitian but not positive definite. Nevertheless, we can apply the above scheme iteratively, replacing the initial system's state by (B4) to get (16).
[1] E. Noether, Invariante Variationsprobleme, Nachrichten von der Gesellschaft der Wissenschaften zu Göttingen, MathematischPhysikalische Klasse 1918, 235 (1918).

[2] M. Peskin and D. Schroeder, An Introduction to Quantum Field Theory (CRC Press, Boca Raton, FL, 2018).

[3] S. D. Bartlett, T. Rudolph, and R. W. Spekkens, Reference frames, superselection rules, and quantum information, Rev. Mod. Phys. 79, 555 (2007).

[4] E. P. Wigner, Die messung quantenmechanischer operatoren, Z. Phys. 131, 101 (1952).

[5] H. Araki and M. M. Yanase, Measurement of quantum mechanical operators, Phys. Rev. 120, 622 (1960).

[6] M. M. Yanase, Optimal measuring apparatus, Phys. Rev. 123, 666 (1961)

[7] J. B. Hartle, R. Laflamme, and D. Marolf, Conservation laws in the quantum mechanics of closed systems, Phys. Rev. D 51, 7007 (1995).

[8] Y. Aharonov, S. Popescu, and D. Rohrlich, On Conservation Laws in Quantum Mechanics, Proc National Acad Sci 118, e1921529118 (2021)

[9] N. Gisin and E. Z. Cruzeiro, Quantum measurements, energy conservation and quantum clocks, Ann. Phys. (Leipzig) 530, 1700388 (2018)

[10] H. Larocque, F. Bouchard, V. Grillo, A. Sit, S. Frabboni, R. E. Dunin-Borkowski, M. J. Padgett, R. W. Boyd, and E. Karimi, Nondestructive Measurement of Orbital Angular Momentum for an Electron Beam Phys. Rev. Lett. 117, 154801 (2016).

[11] S. M. Lloyd, M. Babiker, G. Thirunavukkarasu, and J. Yuan, Electron vortices: Beams with orbital angular momentum, Rev. Mod. Phys. 89, 035004 (2017).

[12] K. Y. Bliokh et al., Theory and applications of free-electron vortex states, Phys. Rep. 690, 1 (2017).

[13] J. V. Koski, V. F. Maisi, T. Sagava, and J. P. Pekola, Experimental Observation of the Role of Mutual Information in the Nonequilibrium Dynamics of a Maxwell Demon, Phys. Rev. Lett. 113, 030601 (2014).

[14] C. Elouard, D. Herrera-Marti, B. Huard, and A. Auffeves, Extracting Work from Quantum Measurement in Maxwell's Demon Engines, Phys. Rev. Lett. 118, 260603 (2017).

[15] M. Naghiloo, J. J. Alonso, A. Romito, E. Lutz, and K. W. Murch, Information Gain and Loss for a Quantum Maxwell's Demon, Phys. Rev. Lett. 121, 030604 (2018).
[16] A. Pais, Einstein and the quantum theory, Rev. Mod. Phys. 51, 863 (1979)

[17] N. D. Mermin, Is the moon there when nobody looks? Reality and the quantum theory, Phys. Today 38(4), 38 (1985).

[18] P. Grangier and A. Auffeves, What is quantum in quantum randomness? Phil. Trans. R. Soc. A 376, 20170322 (2018).

[19] A. Peres, Quantum Theory-Concept and Methods (Kluwer, Dordrecht, The Netherlands, 2002)

[20] Y. Aharonov, D. Z. Albert, and L. Vaidman, How the Result of a Measurement of a Component of the Spin of a Spin-1/2 Particle Can Turn Out to be 100, Phys. Rev. Lett. 60, 1351 (1988).

[21] A. J. Leggett and A. Garg, Quantum Mechanics versus Macroscopic Realism: Is The Flux There when Nobody Looks? Phys. Rev. Lett. 54, 857 (1985).

[22] C. Emary, N. Lambert, and F. Nori, Leggett-Garg inequalities, Rep. Prog. Phys. 77, 016001 (2014).

[23] A. Palacios-Laloy, F. Mallet, F. Nguyen, P. Bertet, D. Vion, D. Esteve, and A. N. Korotkov, Experimental violation of a Bell's inequality in time with weak measurement, Nat. Phys. 6, 442 (2010).

[24] A. J. Leggett, Comment on How the Result of a Measurement of a Component of the Spin of a Spin-1/2 Particle Can Turn Out to be 100, Phys. Rev. Lett. 62, 2325 (1989).

[25] A. Matzkin, Weak values and quantum properties, Found. Phys. 49, 298 (2019).

[26] A. Bednorz, K. Franke, and W. Belzig, Noninvasiveness and time symmetry of weak measurements, New J. Phys. 15, 023043 (2013).

[27] M. Neumark, Spectral functions of a symmetric operator, Izv. Akad. Nauk SSSR Ser. Mat. 4, 277 (1940).

[28] L. Plimak and S. Stenholm, Causal signal transmission by quantum fields. V: Generalised Keldysh rotations and electromagnetic response of the Dirac sea, Ann. Phys. (NY) 327, 2691 (2012).

[29] A. Bednorz and W. Belzig, Quasiprobabilistic Interpretation of Weak Measurements in Mesoscopic Junctions, Phys. Rev. Lett. 105, 106803 (2010).

[30] D. Curic, M. C. Richardson, G. S. Thekkadath, J. Florez, L. Giner, and J. S. Lundeen, Experimental investigation of measurement-induced disturbance and time symmetry in quantum physics, Phys. Rev. A 97, 042128 (2018).

[31] A. Bednorz and W. Belzig, Models of mesoscopic time-resolved current detection, Phys. Rev. B 81, 125112 (2010). 
[32] S. Mal, D. Das, and D. Home, Quantum mechanical violation of macrorealism for large spin and its robustness against coarsegrained measurements, Phys. Rev. A 94, 062117 (2016).

[33] S. Kumari and A. K. Pan, Probing various formulations of macrorealism for unsharp quantum measurements, Phys. Rev. A 96, 042107 (2017).

[34] A. Bednorz, W. Bednorz, and W. Belzig, Testing locality and noncontextuality with the lowest moments, Phys. Rev. A 89, 022125 (2014).
[35] J. Bülte, A. Bednorz, C. Bruder, and W. Belzig, Noninvasive Quantum Measurement of Arbitrary Operator Order by Engineered Non-Markovian Detectors, Phys. Rev. Lett. 120, 140407 (2018).

[36] R. F. Streater and A. S. Wightman, PCT, Spin and Statistics, and All that (Princeton University Press, Princeton, NJ, 2000).

[37] A. Bednorz, Relativistic invariance of the vacuum, Eur. Phys. J. C 73, 2654 (2013). 